\title{
Hallazgos Histopatológicos en el Sistema Nervioso Central de Rattus norvegicus Infectados con Angiostrongylus cantonensis
}

\author{
Histopathological Findings in the Central Nervous System of Rattus \\ norvegicus INFECTED WITH Angiostrongylus cantonensis
}

\author{
Luis Solórzano-Alava ${ }^{1,2}$, Francisco Sánchez-Amador ${ }^{1,3}$, Carolina Pérez $^{1,4}$
}

\section{Resumen}

\begin{abstract}
Rattus norvegicus es el huésped definitivo del nematodo Angiostrongylus cantonensis. El objetivo del estudio fue determinar las alteraciones macroscópicas y lesiones histopatológicas en el sistema nervioso central (SNC) de ratas infectadas con $A$. cantonensis. Se trabajó con 35 R. norvegicus. De estas, 23 fueron infectadas experimentalmente con larvas L 3 y 5 ratas quedaron sin inocular (controles), ambos grupos procedentes de bioterio, y 7 ratas capturadas en la ciudad de Guayaquil, Ecuador, cuya infección natural fue comprobada en el laboratorio. Siete de 23 ratas experimentalmente infectadas presentaron cambios de comportamiento (carreras en círculo) a partir del 29 día pos-infección (dpi). Tres de estas ratas fueron sacrificadas a los 30-33 dpi y las otras 4 a los 48-105 dpi; asimismo, todas las ratas infectadas experimentalmente y las capturadas fueron sacrificadas. Los encéfalos fueron extraídos, pesados, preservados con formol al $10 \%$ e histológicamente procesados. En las tres ratas con 30-33 dpi se encontró edema cerebral, gliosis, congestión circular, hiperplasia de células endoteliales y desarrollo larval (L4-L5). En los cerebros de las ratas con más de 48 dpi se encontraron alteraciones similares y dilatación de vasos sanguíneos. El peso del cerebro de ratas positivas a $A$. cantonensis a los 33 y $>48$ dpi fue de 2.02 y $2.03 \mathrm{~g}$, respectivamente, mientras que el peso promedio en ejemplares sanos (controles) fue de $1.82 \mathrm{~g}$. Se concluye que $A$. cantonensis causa lesiones en el SNC de $R$. norvegicus.
\end{abstract}

Palabras clave: Angiostrongylus cantonensis, Rattus norvegicus, edema, gliosis

${ }^{1}$ Instituto Nacional Salud Pública e Investigación (INSPI) Leopoldo Izquieta Pérez, Guayaquil, Ecuador

${ }^{2}$ E-mail: lsolorzano@inspi.gob.ec

${ }_{3}^{3}$ E-mail: fisanchez@inspi.gob.ec

Recibido: 31 de marzo de 2016

Aceptado para publicación: 30 de julio de 2016 
Rattus norvegicus is the definitive host of Angiostrongylus cantonensis. The aim of this study was to determine the type of macroscopic alterations and histopathological findings in the central nervous system(CNS) of $R$. norvegicus infected with $A$. cantonensis. A total of 35 rats were used. Among them, 23 rats from an animal facility were experimentally infected with L3 larvae and 5 remained as controls (not infected), and 7 rats that were captured in the city of Guayaquil, Ecuador, whose natural infection was confirmed in the laboratory. Seven out of 23 artificially infected rats showed changes in behavior (walking in circles) after 29 days post infection (dpi). Three of them were euthanized at 30-33 dpi and the other four at 48-105 dpi; also, all experimental rats and the seven captured rats were euthanized. The brains were dissected, weighed, perfused with $10 \%$ formalin and histologically processed. In the 30-33 dpi rats were found brain edema, gliosis, congestion, circular endothelial cell hyperplasia and development of L4-L5 larvae. In the brains after $48 \mathrm{dpi}$ similar findings and dilation of blood vessels were observed. The average weight of brains positive to $A$. cantonensis at 30-33 and $>48$ dpi was 2.02 and 2.03 g respectively, while in the controls was $1.82 \mathrm{~g}$. It is concluded that $A$. cantonensis causes lesions in the CNS of Rattus norvegicus.

Key words: Angiostrongylus cantonensis, Rattus norvegicus, edema, gliosis

\section{INTRODUCCIÓN}

Angiostrongylus cantonensis fue descrito por primera vez en China en 1935 en los pulmones de Rattus rattus y Rattus norvegicus y reportado como Pulmonema cantonensis (Chen, 1935).

$R$. rattus y $R$. norvegicus son los huéspedes definitivos de $A$. cantonensis. El parásito adulto se localiza en las arterias pulmonares, donde producen larvas L1 que son eliminadas con las heces, las cuales son ingeridas por un molusco que constituye su huésped intermediario (en Ecuador son Achatina fulica y Pomacea spp). Las L1 se convierten en larvas L2-L3, que son ingeridas por los roedores (Rattus spp). Estas larvas llegan al cerebro como L4-L5 a las dos semanas pos-infección, para descender cuatro semanas más tarde al sistema cardiopulmonar, donde alcanza su estado adulto (Alicata, 1991; Dorta-Contrera et al., 2007; Solórzano et al., 2013).
El primer registro de $A$. cantonensis en roedores en las Américas fue en Cuba, donde se encontraron en 12 de 20 ratas $(R$. norvegicus) en seis comunidades de La Habana (Aguiar et al., 1981). Posteriormente se encontró en 20/94 $R$. norvegicus en Louisiana, EEUU (Campbel y Little, 1988) y en años más recientes en animales de vida silvestre en la misma región (Kim et al., 2002), así como en animales domésticos (Costa et al., 2000) y en primates no humanos (Duffy et al., 2004). Wang et al. (2008) señalaron que roedores capturados en Cuba y la República Dominicana presentaban una alta prevalencia de A. cantonensis. Asimismo, se han reportado roedores con angiostrongilosis en Puerto Rico (Andersen et al., 1986), República Dominicana (Vargas, 1992), Jamaica (Lindo et al., 2002), Haití (Raccurt et al., 2003), Brasil (Caldeira et al., 2007) y Ecuador (Martini et al., 2013).

Debido a los reportes en la región y a la escasez de estudios que especifiquen los daños causados por el paso de $A$. cantonensis en el sistema nervioso central, el objetivo del 
presente estudio fue determinar las alteraciones y lesiones anátomo-patológicas que pudieran presentarse en el Sistema Nervioso Central (SNC) y el tiempo que puedan perdurar estos cambios en las ratas silvestres y de laboratorio experimentalmente infectadas.

\section{Materiales y Métodos}

El estudio se llevó a cabo en el Centro de Referencia Nacional de Parasitología del Instituto Nacional de Salud Pública e Investigación (INSPI) Leopoldo Izquieta Pérez, Guayaquil, entre marzo de 2014 y febrero de 2016.

Se utilizaron 28 ejemplares de ratas $R$. norvegicus adultas albinas machos de 30 días de edad, procedentes del bioterio del INSPI. Cinco de ellas se usaron como modelo de ratas sanas (control, sin inoculación) y las 23 restantes conformaron el grupo experimental. Estos animales fueron inoculados $\operatorname{con} A$. cantonensis (104 larvas L3 por roedor), obtenidos a partir de la digestión de caracoles Achatina fulica infectados de la localidad La Troncal, Ecuador. Adicionalmente, se evaluaron siete ratas pardas ( $R$. norvegicus) infectadas naturalmente con $A$. cantonensis, con más de 48 días pos-infección (dpi) y con estadios adultos en sus pulmones, que fueron capturadas en la ciudad de Guayaquil. Infecciones mayores de 48 días fueron constatadas porque las ratas eliminaban larvas L1 en las heces. Los cambios morfológicos del parásito $A$. cantonensis durante su ciclo de vida fueron comparados e identificados según los trabajos descriptivos de Cowie (2013) y Thiengo et al. (2010).

En la captura de las ratas silvestres se usaron 9 trampas Tomahawk $(15 \times 15 \times 45$ $\mathrm{cm})$, que se colocaron estratégicamente en lugares donde había indicios de presencia de roedores, tales como heces, materiales roí- dos y rincones oscuros. Las trampas fueron armadas y ubicadas durante la noche y revisadas temprano en la mañana siguiente (Ibáñez et al., 2012). Se les hizo exámenes de heces y observación de comportamiento (08:00-09:00 y 15:00-16:00) durante 5 días. Las ratas fueron sacrificadas con $\mathrm{CO}_{2}$ (Moreira et al., 2013).

Durante el experimento, los animales recibieron alimento y agua ad libitum y se mantuvieron en ciclos de luz/oscuridad de 12 horas (Caldeira et al., 2007; Ceruti et al., 2010; Martini et al., 2013). Posterior a la inoculación, fueron inspeccionadas todos los días laborables (309 días laborables), tanto por las mañanas (08:00-09:00) como por las tardes (15:00-16:00), anotando cualquier cambio de comportamiento que pudieran ocasionar los parásitos a lo largo de su ciclo de vida. El estudio contó con los permisos para el uso de roedores del bioterio del INSPI-Veterinaria, Guayaquil y se utilizó la «Guía de manejo y cuidado de animales de laboratorio» del Ministerio de Salud de Perú (2008).

Las ratas de laboratorio fueron sacrificadas entre los 30-33 días y entre 48-105 dpi, periodos relacionados con el nivel de desarrollo de las larvas de $A$. cantonensis en el encéfalo; en tanto que las ratas de laboratorio fueron sacrificadas al término de los cinco días de observación. El sacrificio de las ratas se realizó en una cámara de $\mathrm{CO}_{2}$ (Moreira et al., 2013). Se colectaron los cerebros según protocolo usado en el CRN de Parasitología (Martini et al., 2013). Los 35 cerebros fueron pesados en una balanza analítica (en miligramos) - Modelo MET AB54S/FACT

Los tejidos del Sistema Nervioso Central (SNC) fueron fijados y conservados en formalina al $10 \%$, incluidos en parafina, cortadas y teñidas con hematoxilina-eosina para su observación, según el procedimientos estándar de Duffy et al. (2004). 


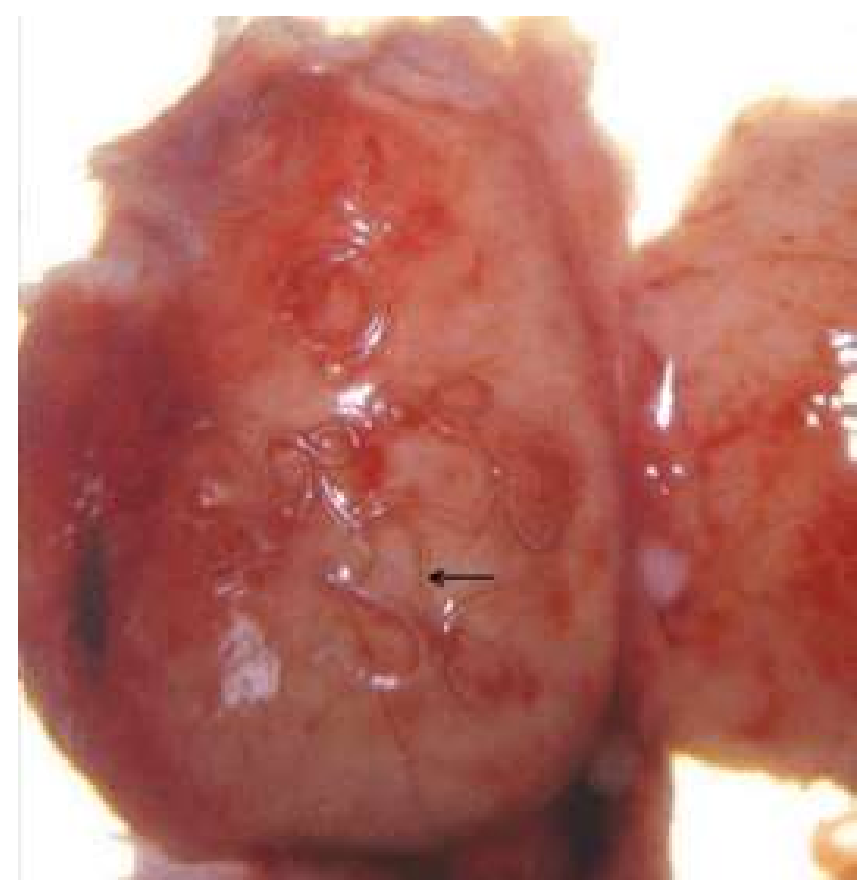

Figura 1. Larvas L4 y L5 de Angiostrongylus cantonensis en el lóbulo izquierdo del cerebro de Rattus norvegicus a los 33 días de la inoculación

\section{Resultados}

Siete de las 23 ratas de laboratorio presentaron cambios temporales de comportamiento (carreras en círculos). Tres de ellas luego de 30-33 dpi y las otras cuatro luego de 48 dpi. Ninguna de las ratas control ni las siete ratas pardas capturadas con infección natural de angiostrongilosis presentaron cambios de comportamiento.

No se observaron alteraciones macroscópicas visibles en el cerebro de las ratas infectadas de manera natural o artificial. No obstante, el peso del cerebro de ratas positivas a A. cantonensis a los $33 \mathrm{y}>48$ dpi varió entre 2.02 y $2.03 \mathrm{~g}$, mientras que el peso promedio en ejemplares sanos (controles) varió entre 1.81 y $1.83 \mathrm{~g}$.

\section{Cerebro a los 30-33 Días Pos-Infección}

En la necropsia de tres de las 23 ratas inoculadas, se observaron larvas vivas L4L5 de $A$. cantonensis en la superficie de los lóbulos cerebrales, interior del cerebelo y adheridas a las meninges del cerebro (Figura 1). En estas ratas se observaron cambios de comportamiento durante tres días (giros en círculos por un intervalo de tiempo).

En la evaluación histopatológica del cerebro se encontró hiperplasia de células endoteliales, congestión circulatoria en arteriolas, gliosis y edema intersticial. Además, se observó dentro del encéfalo, en las meninges y en el cerebelo, cortes de larvas que corresponden al esófago, intestino y órganos sexuales inmaduros de larvas L4-L5 de $A$. cantonensis. 


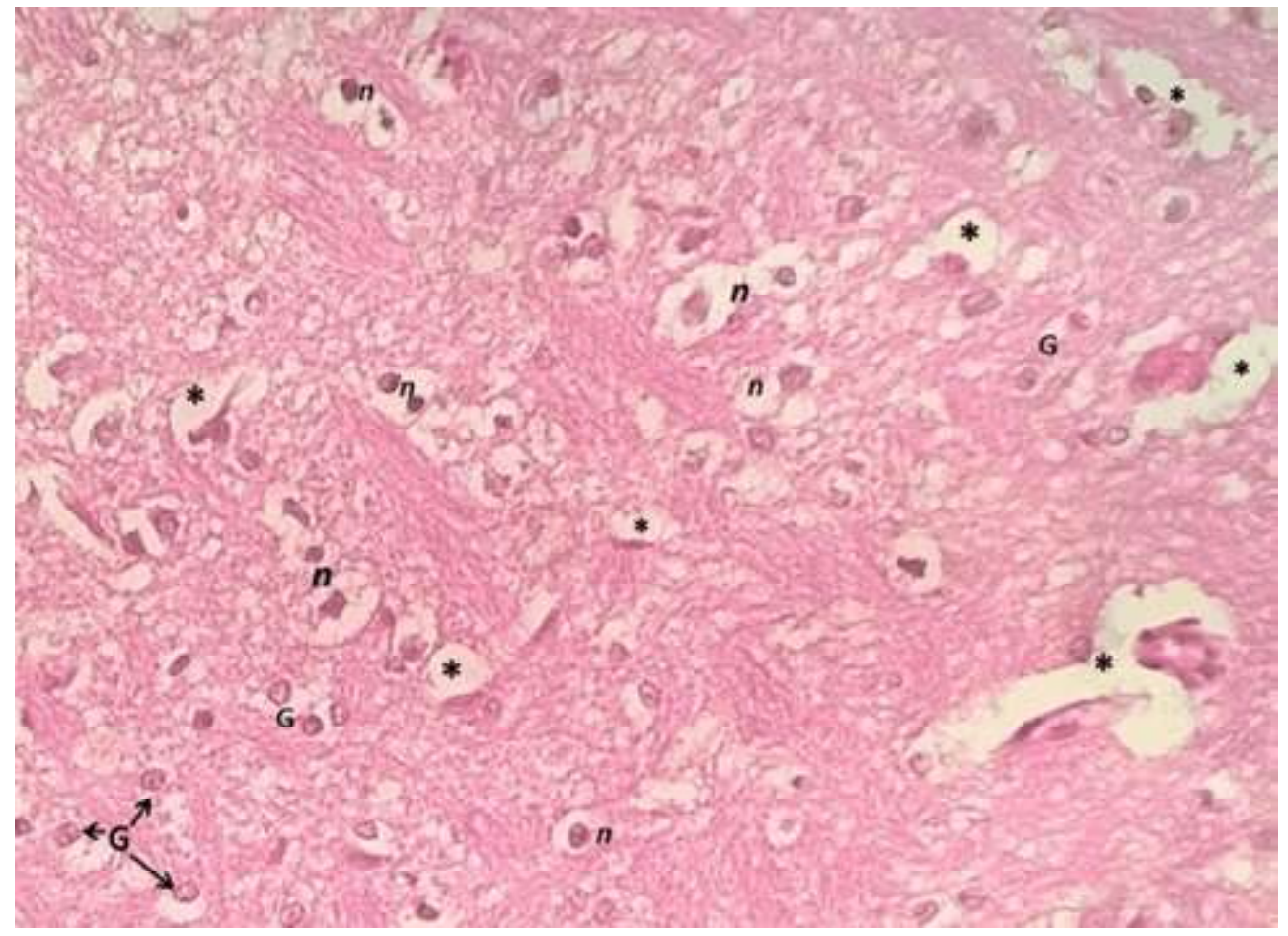

Figura 2. Presencia de edema vacular $(*)$, células gliales $(\mathrm{G})$ y neuronas (n) en el parénquima cerebral de Rattus norvegicus con más de 48 días de infección experimental con Angiostrongylus cantonensis

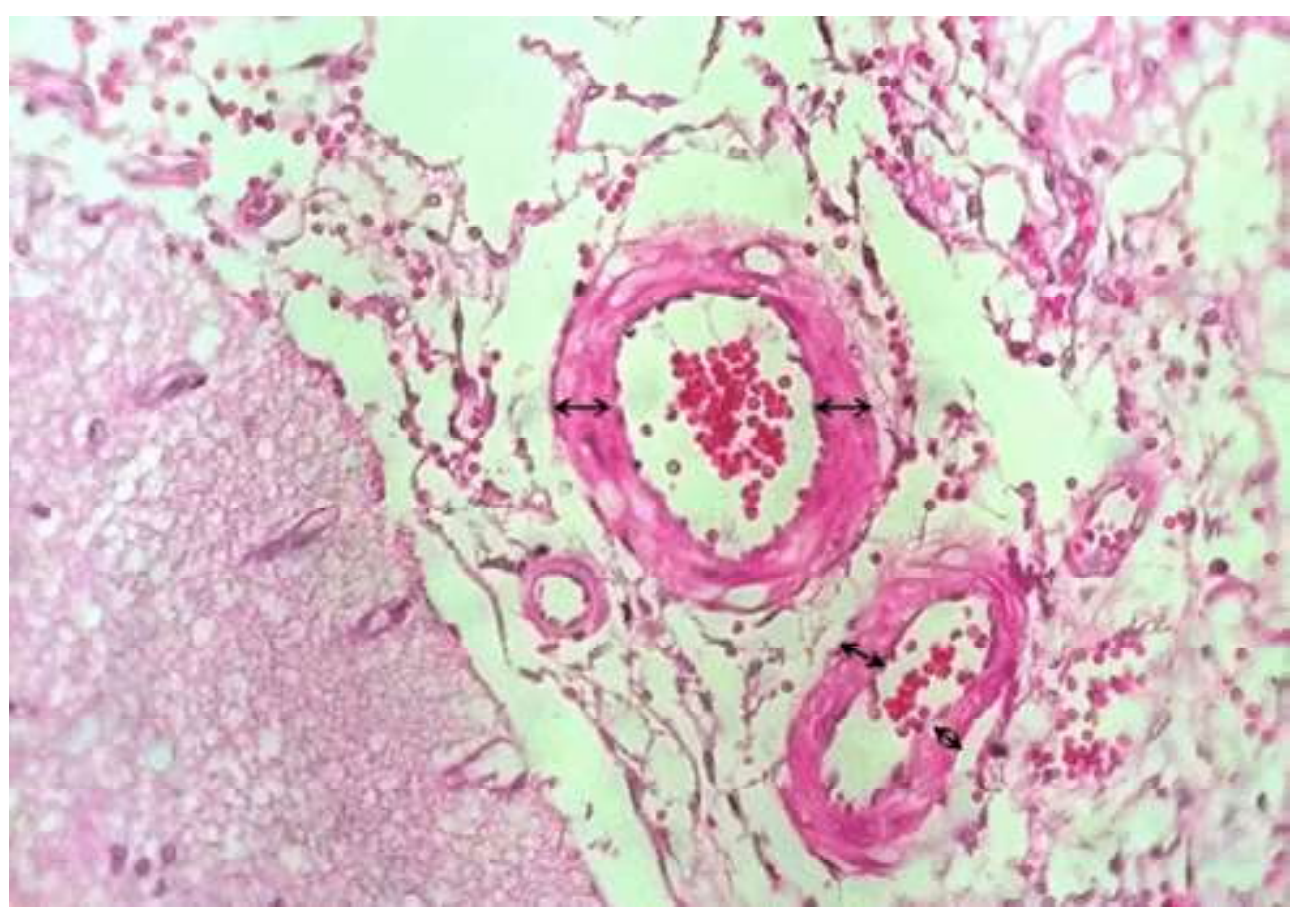

Figura 3. Arteriolas cerebrales dilatadas (flechas) con glóbulos rojos en parénquima cerebral de Rattus norvegicus con más de 48 días de infección experimental con Angiostrongylus cantonensis 


\section{Cerebro Posterior a los 48 Días Pos-In- fección}

En las 20 ratas restantes con angiostrongilosis (larvas L1 en heces), solo cuatro presentaron cambios de comportamiento los días 48, 49, 58 y 105 dpi, respectivamente. Las 16 ratas restantes fueron sacrificadas a los 48 (2), 49 (2), 58 (3), 60 (3), 90 (4) y 105 (2) dpi. No obstante, en la evaluación histopatológica de las 20 ratas se encontró hiperplasia de células endoteliales, gliosis y edema intersticial. Además, dilatación en los vasos sanguíneos encefálicos, creando una congestión vascular cerebral más severa que la observada en las ratas infectadas a los 30-33 dpi.

En las siete ratas pardas capturadas (positivas a angiostrongilosis con parásitos adultos en arterias pulmonares) no se encontraron parásitos en el SNC. Por otro lado, a nivel histológico se encontraron alteraciones similares a las observadas en las 23 ratas infectadas experimentalmente con larvas L1.

\section{Evaluación en Ratas Control}

En la evaluación macroscópica e histopatológica de los encéfalos de ratas sanas ( $\sin$ inocular), no se encontraron cambios patológicos evidentes ni presencia de otros tipos de parásitos.

\section{Discusión}

La presencia de larvas L4-L5 en la bóveda cefálica a los 33 dpi, de parásitos adultos en los pulmones posterior a los 48 dpi y larvas L1 en heces concuerda con trabajos previos de los autores (Solórzano et al., 2013).

Wang et al. (2008) no observaron cambios evidentes a los 7 dpi, pero encontraron algunas células inflamatorias que se incrementaron en las meninges a los 14 dpi y otras aparecieron en el cerebro después de
21 dpi en ratas infectadas con $A$. cantonensis. Asimismo, Wei et al. (2015) demostraron que los ratones y las ratas infectadas con A. cantonensis exhiben síntomas conductuales evidentes del SNC, aunque leves, y desaparecen a las tres semanas de la infección. Es posible que las diferencias con el presente estudio estén relacionadas a una diferente respuesta inmune, pues los cambios de comportamiento fueron observados en los 48, 49, 56 y 60 dpi.

El mayor peso de cerebros de ratas con angiostrongilosis en comparación con animales sanos pudo deberse al edema e inflamación (hiperplasia intersticial) que no permite recuperar el peso inicial. Yoshimura et al. (1988) indicaron que ratones y cobayos infectados mostraron atrofia degenerativa o pérdida de células de Purkinje y vacuolización esponjosa de la materia blanca en el cerebelo.

Las microglías pueden tener un impacto en la respuesta inflamatoria del SNC y mejorar la función de los nervios del SNC en la angiostrongiliasis (Wei et al., 2013). Similares resultados de edemas y gliosis fueron encontrados en la evaluación histopatológica en 14 ratas ( $R$. norvegicus) con angiostrongilosis de 30-33 y $>48$ dpi.

Caldeira et al. (2007) reportaron la presencia de $A$. cantonensis jóvenes en las venas meníngeas de $R$. norvegicus experimentalmente infectados. Además, observaron dilatación de venas y arterias por el paso del parásito. En el presente estudio solo se encontraron arteriolas dilatadas en ratas posteriores a los $48 \mathrm{dpi}$, pero sin presencia del parásito.

Experiencias previas en el laboratorio de los autores indican que ratas inoculadas que poseen signos de alteraciones neurológicas y que mueren espontáneamente a los 30-33 dpi se les ha encontrado entre 20 y 50 larvas L4-L5 de A. cantonensis en el SNC durante la necropsia. Asimismo, se ha encontrado ratas con más de un año postinoculación donde se han recuperado hasta 
105 parásitos adultos en las arterias pulmonares, por lo que resulta difícil establecer la carga parasitaria necesaria para producir la muerte del huésped definitivo $(R$. norvegicus). Las ratas poseen una resistencia natural propia contra las enfermedades parasitarias (Landete y Barja, 1998).

\section{Conclusiones}

- Se observaron cambios de comportamiento (pérdida de orientación temporal) en $7 / 23$ ratas (Rattus norvegicus) a partir del día 29 de la infección experimental con Angiostrongylus cantonensis.

- Se observó la presencia del parásito en las meninges, pero sin cambios macroscópicos de la morfología del cerebro; sin embargo, hubo diferencias en el peso del cerebro de animales infectados vs. ratas controles.

- Se observó edema, hiperplasia de células endoteliales y gliosis a los 30-33 y 48 días pos-infección y dilatación de las arteriolas cerebrales posterior a los 48 días pos-infección.

\section{Agradecimientos}

Los autores expresan su agradecimiento a los integrantes del laboratorio del Centro de Referencia Nacional de Parasitología y Plataforma de Anatomía Patológica del Instituto Nacional de Investigación en Salud Pública (INSPI), Guayaquil, por el apoyo prestado. Asimismo, al tecnólogo Juan Torres y a la Sra. Gladys Marlene Amador Palma por la corrección y realización de este escrito. El estudio fue financiado por el INSPI-Guayaquil y por los propios autores del estudio.

\section{Literatura Citada}

1. Aguiar PH, Morera P. Pascual J. 1981. Primer registro de Angiostrongylus cantonensis en Cuba. Am J Trop Med Hyg 30: 963-965.
2. Alicata JE. 1991. The discovery of Angiostrongylus cantonensis as a cause of human eosinophilic meningitis. Parasitol Today 7: 151-153.

3. Andersen E, Gubler DJ, Sorensen K, Beddard J, Ash LR. 1986. First report of Angiostrongylus cantonensis in Puerto Rico. Am J Trop Med Hyg 35: 319-322.

4. Caldeira R, Mendonça C, Oliveira $C$, Lenzi H, Graeff-Teixeira C, Lima W, Mota E, Lea I, et al. 2007. First record of molluscs naturally infected with Angiostrongylus cantonensis (Chen, 1935) (Nematoda: Metastrongylidae) in Brazil. Mem Inst Oswaldo Cruz 102: 887-889. doi: 10.1590/S0074-02762007000700018

5. Campbell BG, Little MD. 1988. The finding of Angiostrongylus cantonensis in rats in New Orleans. Am J Trop Med Hyg 38: 568-573.

6. Chen TH. 1935. Un nouveau nemátode pulmonairé: Pulmonema cantonensis $\mathrm{n}$. g. n. sp., de rats de Canton. Ann Parasitol Hum Comp 13: 321-327.

7. Costa R, McClure J, Snider G, Stewart B. 2000. Verminous meningoencephalomyelitis by Angiostrongylus (Parastrongylus) cantonensis in an American miniature horse. Eq Vet Educ 12: 2-6. doi: 10.1111/j.2042-3292.2000. tb01754.x

8. Cowie HR. 2013. Biology, systematics, life cycle, and distribution of Angiostrongylus cantonensis, the cause of rat lungworm disease. Hawaii $\mathrm{J}$ Med Public Health 72(2): 6-9.

9. Dorta-Contreras A, Núñez F, Pérez O, Lastre M, Magraner M, Bu-Coifiú, Noris E, et al. 2007. Peculiaridades de la meningoencefalitis por Angiostrongylus cantonensis en América. Rev Neurol 45: 755-763.

10. Duffy SM, Miller C, Kinsella M, Lahunta A. 2004. Parastrongylus cantonensis in nonhuman primate, Florida. Emerg Infect Dis 10: 2207-2210. doi: 10.3201/eid1012.040319 
11. Ibáñez CA, Berovides V, Hernández N, Cantillo J, De la Fuente J. 2012. Relación entre la morfofisiología y la carga de ectoparásitos en dos poblaciones de ratas (Rattus sp.) de La Habana. Rev Cubana Med Trop 64: 224-234.

12. Kim D, Stewart T, Bauer W, Mitchell M. 2002. Parastrongylus (=Angiostrongylus) cantonensis now endemic in Louisiana wildlife. J Parasitol 88: 10241026. doi: 10.1645/0022-3395(2002) 088[1024:PACNEI]2.0.CO;2

13. Landete CT, Barja CA. 1998. La rata de alcantarilla (Rattus norvegicus). Ecología, comportamiento y control. España: Univ de Castilla-La Mancha. 56 p.

14. Lindo JF, Waugh $C$, Hall J, Cunningham-Myrie C, Ashley D, Mark L, Eberhard M, et al. 2002. Enzootic Angiostrongylus cantonensis in rats and snails after an outbreak of human eosinophilic meningitis, Jamaica. Emerg Infect Dis 8: 324-326.

15. Martíni RL, Gómez E, Muzzio J, Solórzano L. 2013. Primer registro de ratas infectadas con Angiostrongylus cantonensis, descripción del primer foco endémico de transmisión natural en Ecuador. En: XXI Congreso Latinoamericano de Parasitología. Guayaquil: Federación Latinoamericana de Parasitología (FLAP).

16. Martini RL, Muzzio AJ, Orlando NA. 2013. Guía operativa para la vigilancia epidemiológica, parasitológica y malacológica de la meningoencefalitis eosinofílica causada por el parásito: Angiostrongylus cantonensis. Guayaquil, Ecuador: Instituto Nacional de Higiene y Medicina Tropical «Dr. Leopoldo Izquieta Pérez». [Internet]. Disponible en: http://luiggimartinirobles.blogspot.co.at/ 2014/04/guia-operativa-para-lavigilancia.html

17. Ministerio de Salud. 2008. Guía de manejo y cuidado de animales de laboratorio: ratón. Lima, Perú: Instituto Nacional de Salud. [Internet]. Disponible en: http:/ /bvs.minsa.gob.pe:81/1ocal/INS/ 962_INS68.pdf
18. Moreira VL, Giese EG, Melo FT, Simões RO, Thiengo SC, Maldonado A Jr, Santos JN. 2013. Endemic angiostrongyliasis in the Brazilian Amazon: natural parasitism of Angiostrongylus cantonensis in Rattus rattus and $R$. norvegicus, and sympatric giant African land snails, Achatina fulica. Acta Trop 125(1): 90-97. doi: 10.1016/ j.actatropica.2012.10.001

19. Raccurt CP, Blaise J, Durette-Desset MC. 2003. Présence d'Angiostrongylus cantonensis en Haiti. Trop Med Int Health 8: 423-426.

20. Solórzano L, Martini L, Hernández AH, Sarracent PJ, Muzzio J, Rojas RL. 2013. Angiostrongylus cantonensis: un parásito emergente en Ecuador. Rev Cubana Med Trop [Internet]. Disponible en: http://www.revmedtropical.sld.cu/index.php/medtropical/ article/view/4/3

21. Thiengo CS, Maldonado A, Mota ME, Torres JLE, Caldeira R, Carvalho SO, Oliveira PMA, et al. 2010. The giant African snail Achatina fulica as natural intermediate host of Angiostrongylus cantonensis in Pernambuco, northeast Brazil. Acta Trop 115: 194-199. doi: 10.1016/j.actatropica.2010.01.005

22. Vargas M, Gómez Pérez JD, Malek EA. 1992. First record of $A$. cantonensis (Chen, 1935) (Nematoda: Metastrongyloidae) in the Dominican Republic. Trop Med Parasitol 43: 253255.

23. Wang $Q$, Lai D, Zhu $X$, Chen $X$, Lun Z. 2008. Human angiostrongyliasis. Lancet Infect Dis 8: 621-630. doi: 10.1016/S1473-3099(08)70229-9

24. Wei J, Wu F, He A, Zeng X, Ouyang L, Liu M, Zheng H, et al. 2015. Microglia activation: one of the checkpoints in the CNS inflammation caused by Angiostrongylus cantonensis infection in rodent model. Parasitol Res 114: 3247-3254. doi: 10.1007/s00436-015-4541-9 
25. Wei J, Wu Feng, Sun Xi, Zeng $X$, Liang J, Zheng $\mathrm{H}, \mathrm{Yu} X$, Zhang $\mathrm{K}$, $\boldsymbol{W u} Z$. 2013. Differences in microglia activation between rats-derived cell and mice-derived cell after stimulating by soluble antigen of IV larva from Angiostrongylus cantonensis in vitro. Parasitol Res 112: 207-214. doi: 10.1007/ s00436-012-3127-z
26. Yoshimura K, Sugaya H, Kawamura K, Kumagai M. 1988. Ultrastructural and morphometric analyses of eosinophils from the cerebrospinal fluid of the mouse and guinea-pig infected with Angiostrongylus cantonensis. Parasite Immunol 10: 411-423. doi: 10.1111/j.13653024.1988.tb00231.x 